\title{
Enhancing Security in Cognitive Radio Multicast Networks Using Interference Power
}

\author{
Rezwana Sultana, Md. Zahurul Islam Sarkar, Md. Selim Hossain \\ Department of Electrical and Electronic Engineering, Rajshahi University of Engineering and Technology, Rajshahi, Bangladesh \\ Email: dola.ruet@gmail.com
}

How to cite this paper: Sultana, R., Sarkar, Md.Z.I. and Hossain, Md.S. (2019) Enhancing Security in Cognitive Radio Multicast Networks Using Interference Power. Journal of Computer and Communications, 7, 66-78.

https://doi.org/10.4236/jcc.2019.712007

Received: November 6, 2019

Accepted: December 13, 2019

Published: December 16, 2019

Copyright $\odot 2019$ by author(s) and Scientific Research Publishing Inc. This work is licensed under the Creative Commons Attribution International License (CC BY 4.0).

http://creativecommons.org/licenses/by/4.0/

\begin{abstract}
In this paper, security issue in multiple input multiple output (MIMO) multicasting system has been analyzed in the presence of a group of eavesdroppers in cognitive radio networks (CRNs). Primary base station (PBS) and secondary base station (SBS) communicate with multiple primary and secondary receivers, respectively via a precoding relay having multiple antennas. At first, considering interference the secrecy multicast capacity at the primary receivers (PRs) and the secondary receivers (SRs) has been calculated and investigated the impact of interferences on it. Then, the zero-forcing (ZF) precoding technique at the relay has been employed which enhances the secrecy multicast capacity at the PRs and SRs by zeroing the impact of interference on each other. Secondly, the existing constructive interference energy of the communication medium employing selective precoding (SP) technique at the relay has been used to improve the secrecy multicast capacity of the PRs and SRs. Finally, phase alignment precoding (PAP) technique at the relay has been introduced which uses the destructive part of interference for further increase in the secrecy multicast capacity at the PRs and SRs. It is observed that among the three precoding techniques, the best performance is achieved by using the PAP at the relay in terms of secrecy multicast capacity and secure outage probability analysis. This is due to the fact that PAP technique at the relay not only uses the constructive interference part but also it rotates the destructive interference part in such a way that the resulting interference is always instantaneously constructive. So using these precoding relays interference power can be used to enhance system performance without increasing base station power.
\end{abstract}

\section{Keywords}

Primary Users, Secondary Users, Cognitive Radio Network, Selective Precoding, Zero-Forcing Precoding, Phase Alignment Precoding and Multicasting 


\section{Introduction}

In modern communication world, researchers have shown their great interest on the study of CRNs due to its dynamic spectrum management technique. Multicasting is a group oriented communication technique where the capacity of the system degrades with the addition of new user. To overcome the problem of capacity degradation in wireless multicasting with the addition of new users to the system can be resolved efficiently by utilizing CRNs. The use of precoding techniques plays an important role in interference cancelation which is a considerable issue in CRNs. Christos et al. in [1] used ZF precoding, SP and PAP for analysing the performance of cognitive relay assisted MIMO downlink channels in the absence of eavesdropper. In [2] authors used ZF and SP linear precoding techniques in enhancing multicast capacity of Heterogeneous networks. Authors used ZF and SP precoding relay to enhance secrecy multicast capacity considering one user in [3]. Relay selection in CRNs has been studied in [4] for secured transmission in the presence of eavesdropper. In [5] authors deal with cheat proof cooperative relay in CRNs. In [6] an achievable secrecy rate of communication for PUs as well as SUs has been studied. Action recognition techniques in the frequency domain have been studied in [7] for detecting PU emulation. In [8] authors maximize the secrecy throughput of the primary user by designing and optimizing the beamforming techniques. In order to improve security, in [9] authors propose a user scheduling scheme to achieve multiuser diversity. In [10], capacity bounds have been investigated for two sources which simultaneously transmit signals to two destinations with the help of a full-duplex relay node. In [11], authors proposed Block Diagonalization based precoding scheme which removes the inter-user interference and then they combined their proposed method with maximal signal to jamming plus noise ratio (SJNR) precoding method to minimize the inter-user interference. In [12], authors propose full-projection (FP) and partial-projection (PP) based precoding for mitigating interference effect in Cognitive radio MIMO network and observed that FP-based precoding scheme avoids of the primary system and PP-based scheme improver the cognitive radio throughput. Ahthors applied IC techniques in cognitive radio network to mitigate the inter network interference and observed that the receivers assisted by proper IC techniques server the purpose provided that the secondary signals have dissimilar characteristics to those of primary signals in [3].

In [2] authors used two precoding techniques (ZF and SP linear precoding techniques) but they did not consider security issue as well as the concept of PAP technique. Again in [1] authors worked with ZF, SP and PAP precoding techniques but the multicasting scenario and security issue are not considered. So considering the importance of secured wireless multicasting, in this paper, the secrecy multicast capacity (SMC) and Secure Outage Probability (SOP) of CRNs for compensating the effect of interferences using ZF precoding at the relay have been characterized. Then, in order to improve the SMC and SOP, we employ SP at the relay. Finally, the PAP technique is used to further enhance the 
SMC and SOP by accommodating the destructive part of interference.

The rest of the paper is organized as follows. The system model and the formulation of problem are discussed in Section 2. In Section 3, we describe the effect of interferences on the SMC without precoding relay. In Section 4, we show, how the enhancement of secrecy capacity can be achieved using ZF precoding, SP and PAP at the relay. SOP and numerical results are analyzed in Section 5 and 6, respectively. Finally, Section 7 describes the concluding remarks of this work.

\section{System Model and Problem Formulation}

We consider a MIMO CRN as shown in Figure 1 in which a PBS and a SBS are communicating with $P$ PRs and $S$ SRs, respectively in the presence of $K$ eavesdroppers. PBS and SBS are equipped with $n_{B_{1}}$ and $n_{B_{2}}$ antennas, respectively while each eavesdroppers is equipped with $n_{E}$ antennas. Each PRs and SRs are equipped with $n_{P}$ and $n_{S}$ antennas, respectively. To limit the joint average transmit power of the network, each PRs and SRs receives a predetermined scaling factor from the precoding relay. The precoding relay is assumed to be equipped with $n_{B_{1}}+n_{B_{2}}$ receive antennas and $n_{R}$ transmit antennas. Using high speed connection between the PRs and SRs and the relay via a backhaul network as described in [13], the receiving antennas of the relay receive channel state information and information about instantaneous data symbols [14].

The direct channel coefficients between PBS and PRs are $\mathbf{H}_{a} \in \mathbb{C}^{n_{P} \times n_{B_{1}}}$ where $a=1, \cdots, P$ and that between SBS and SRs are $\mathbf{B}_{b} \in \mathbb{C}^{n_{S} \times n_{B_{2}}}$ where $b=1, \cdots, S$. The cross-over channel coefficients between PBS and SRs are $\mathbf{D}_{b} \in \mathbb{C}^{n_{S} \times n_{B_{1}}}$ and

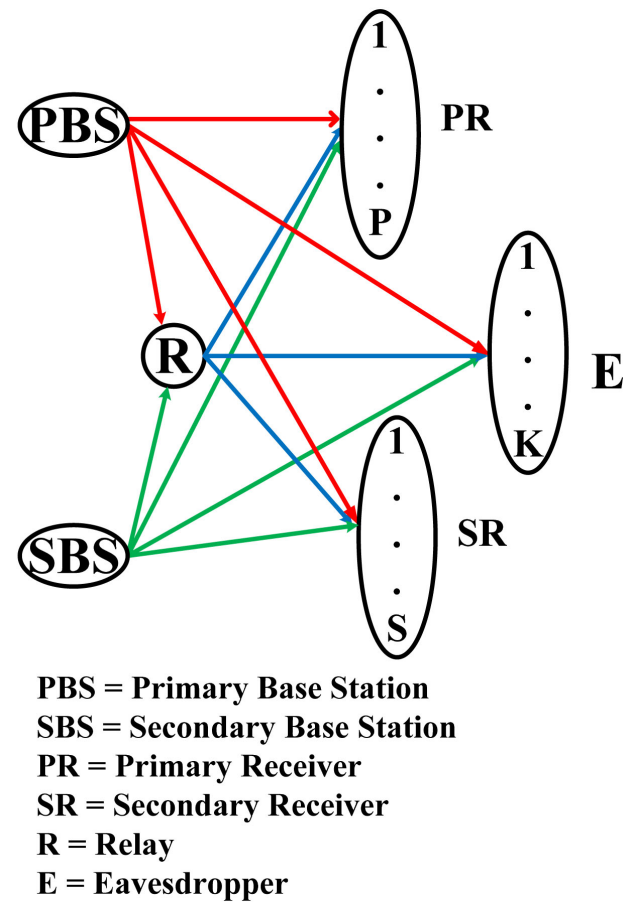

Figure 1. System model. 
that between SBS and PRs are $\mathbf{G}_{a} \in \mathbb{C}^{n_{P} \times n_{B_{2}}}$. The channel coefficients between PBS and eavesdroppers are $\mathbf{I}_{e} \in \mathbb{C}^{n_{E} \times n_{B_{1}}}$, where $e=1, \cdots, K$ and that between SBS and eavesdroppers are $\mathbf{J}_{e} \in \mathbb{C}^{n_{E} \times n_{B_{2}}}$. All the channels are assumed to be Rayleigh fading and the powers assigned to the PBS, SBS and the relay are denoted by $P_{P}, P_{S}$ and $P_{R}$, respectively.

In the following sections, at first, we describe direct transmission scheme without relay to quantify the impact of interference on the SMC at the PRs and SRs. Then, we nullify the effect of interferences employing ZF precoding at the relay. After that, to use the existing constructive part of interference as an additional source of green energy, SP at the relay has been introduced which enhances the SMC and SOP. Finally, by rotating the correlation angle of the interference, we enhance the SMC and SOP employing PAP at the relay.

\section{Transmission without Precoding Relay}

The transmitted signals of SBS act as interference at the PRs and eavesdroppers in the absence of the precoding relay. Similar situation is considered for SRs. Hence, the received signals at the ath PRs, $b$ th SRs and eth eavesdroppers are respectively given by

$$
\begin{aligned}
& \mathbf{y}_{P_{a}}=\mathbf{H}_{a} \mathbf{x}_{1}+\underbrace{\mathbf{G}_{a} \mathbf{x}_{2}+\mathbf{n}_{P_{a}}}_{\mathbf{w}_{a}}, \\
& \mathbf{y}_{S_{b}}=\mathbf{B}_{b} \mathbf{x}_{2}+\underbrace{\mathbf{D}_{b} \mathbf{x}_{1}+\mathbf{n}_{S_{b}}}_{\mathbf{w}_{b}}, \\
& \mathbf{y}_{E_{e}}=\mathbf{I}_{e} \mathbf{x}_{1}+\underbrace{\mathbf{J}_{e} \mathbf{x}_{2}+\mathbf{n}_{E_{e}}}_{\mathbf{w}_{e}},
\end{aligned}
$$

where $\mathbf{x}_{1} \in \mathbb{C}^{n_{B_{1}} \times 1}$ and $\mathbf{x}_{2} \in \mathbb{C}^{n_{B_{2}} \times 1}$ denote the transmitted signal of PBS and SBS, respectively. $\mathbf{n}_{P_{a}} \sim \mathcal{C N}\left(0, N_{P} \mathbf{I}_{n_{P}}\right), \mathbf{n}_{S_{b}} \sim \mathcal{C N}\left(0, N_{S} \mathbf{I}_{n_{S}}\right)$ and $\mathbf{z}_{e_{k}} \sim \mathcal{C N}\left(0, N_{E} \mathbf{I}_{n_{E}}\right)$ denote the Gaussian noises imposed on the ath PRs, $b$ th SRs and eth eavesdroppers, respectively. $\mathbf{w}_{a}, \mathbf{w}_{b}$ and $\mathbf{w}_{e}$ denote interference plus noise at the ath PRs, $b$ th SRs and eth eavesdropper, respectively.

The mutual information at the ath PRs is given by

$$
I\left(\mathbf{x}_{1} ; \mathbf{y}_{P_{a}} \mid \Theta_{a}\right)=h\left(\mathbf{y}_{P_{a}} \mid \Theta_{a}\right)-h\left(\mathbf{w}_{a} \mid \mathbf{G}_{a}\right),
$$

where, $\Theta_{a}=\left[\begin{array}{ll}\mathbf{H}_{a} & \mathbf{G}_{a}\end{array}\right] . h(\cdot)$ denotes entropy of a function. Defining

$\mathbf{Q}_{x}=\mathbb{E}\left\{\mathbf{x}_{1} \mathbf{x}_{1}^{\dagger}\right\}=\frac{P_{P}}{n_{B_{1}}} \mathbf{I}_{B_{B_{1}}}$ as covariance of $\mathbf{x}_{1}$ and $\mathbf{Q}_{s}=\mathbb{E}\left\{\mathbf{x}_{2} \mathbf{x}_{2}^{\dagger}\right\}=\frac{P_{S}}{n_{B_{2}}} \mathbf{I}_{B_{B_{2}}}$ as covariance of $\mathbf{x}_{2}$, the covariance of received signal at the ath PRs, $\mathbf{y}_{P_{a}}$ is expressed as

$$
\mathbf{Q}_{y_{P_{a}}}=\mathbb{E}\left(\mathbf{y}_{P_{a}} \mathbf{y}_{P_{a}}^{\dagger}\right)=N_{P}\left[\mathbf{I}_{n_{P}}+\Theta_{a} \Lambda_{1} \Theta_{a}^{\dagger}\right],
$$

where $\mathbb{E}\{\cdot\}$ and $(\cdot)^{\dagger}$ denote the expectation and conjugate transpose operations, respectively and $\Lambda_{1}=\frac{1}{N_{P}} \mathbf{Q}_{x_{1}} \oplus \frac{1}{N_{P}} \mathbf{Q}_{x_{2}}=\frac{P_{P}}{n_{B_{1}} N_{P}} \oplus \frac{P_{S}}{n_{B_{2}} N_{P}}$. Hence, the 
entropy of $\mathbf{y}_{P_{a}}$ is given by

$$
h\left(\mathbf{y}_{P_{a}} \mid \Theta_{a}\right)=\log _{2} \operatorname{det}\left[\pi e N_{P}\left(\mathbf{I}_{n_{P}}+\Theta_{a} \Lambda_{1} \Theta_{a}^{\dagger}\right)\right] .
$$

Similarly, the entropy of $\mathbf{w}_{a}$ is expressed as

$$
h\left(\mathbf{w}_{a} \mid \mathbf{G}_{a}\right)=\log _{2} \operatorname{det}\left[\pi e N_{P B S}\left(\mathbf{I}_{n_{P}}+\frac{P_{S}}{n_{B_{2}} N_{P}} \mathfrak{G}\right)\right],
$$

where $\mathfrak{G}=\mathbf{G}_{a} \mathbf{G}_{a}^{\dagger}$. Therefore, substituting the values of $h\left(\mathbf{y}_{P_{a}} \mid \Theta_{a}\right)$ and $h\left(\mathbf{w}_{a} \mid \mathbf{G}_{a}\right)$ in Equation (4), the mutual information at the ath PRs can be written as

$$
I\left(\mathbf{x} ; \mathbf{y}_{P_{a}} \mid \Theta_{a}\right)=\log _{2}\left(\frac{\mathbf{I}_{n_{P}}+\Theta_{a} \Lambda_{1} \Theta_{a}^{\dagger}}{\mathbf{I}_{n_{P}}+\frac{P_{S}}{n_{B_{2}} N_{P}} \mathfrak{G}}\right) .
$$

Hence, the capacity at the ath PRs with interference is given by

$$
C_{P_{a}}=\max _{\Lambda_{1}} \log _{2}\left(\frac{\mathbf{I}_{n_{P}}+\Theta_{a} \Lambda_{1} \Theta_{a}^{\dagger}}{\mathbf{I}_{n_{P}}+\frac{P_{S}}{n_{B_{2}} N_{P}} \mathfrak{G}}\right) .
$$

Now multicast capacity at the $P$ PRs is expressed as

$$
C_{P_{\text {mcast }}}=\max _{\Lambda_{1}} \log _{2}\left(\frac{\mathbf{I}_{n_{P}}+\min _{1 \leq a \leq P} \Theta_{a} \Lambda_{1} \Theta_{a}^{\dagger}}{\mathbf{I}_{n_{P}}+\frac{P_{S}}{n_{B_{2}} N_{P}} \min _{1 \leq a \leq P} \mathfrak{G}}\right) .
$$

Similarly, capacity at the eth eavesdropper is given by

$$
C_{E_{e}}=\max _{\Lambda_{2}} \log _{2}\left(\frac{\mathbf{I}_{n_{E}}+\Omega_{e} \Lambda_{2} \Omega_{e}^{\dagger}}{\mathbf{I}_{n_{E}}+\frac{P_{S}}{n_{B_{2}} N_{P}} \mathbf{J}_{e} \mathbf{J}_{e}^{\dagger}}\right)
$$

where $\Lambda_{2}=\frac{P_{P}}{n_{B_{1}} N_{E}} \oplus \frac{P_{S}}{n_{B_{2}} N_{E}}$ and $\Omega_{e}=\left[\begin{array}{ll}\mathbf{I}_{e} & \mathbf{J}_{e}\end{array}\right]$. Hence, multicast capacity at the $\mathrm{K}$ eavesdroppers can be written as

$$
C_{E_{\text {mcast }}}=\max _{\Lambda_{2}} \log _{2}\left(\frac{\mathbf{I}_{n_{E}}+\max _{1 \leq e \leq K} \Omega_{e} \Lambda_{2} \Omega_{e}^{\dagger}}{\mathbf{I}_{n_{E}}+\frac{P_{S}}{n_{B_{2}} N_{P}} \max _{1 \leq e \leq K} \mathbf{J}_{e} \mathbf{J}_{e}^{\dagger}}\right) .
$$

Therefore, the SMC at the PRs is given by

$$
C_{P_{\text {mcast }}}^{\text {Sec }}=\max _{\mathbf{Q}_{x}} \log _{2}\left[\left(C_{P_{\text {mcast }}}-C_{E_{\text {mcast }}}\right)\right] \text {. }
$$

Using (7) and (9) into (10), the SMC at the PRs is found which is shown in equation (11) at the bottom of first page. Following the similar procedure of deriv- 
ing Equation (11), the SMC at the SRs with interference can be derived which is given at the bottom of next page in Equation (12), where $\alpha_{b}=\left[\begin{array}{ll}\mathbf{B}_{b} & \mathbf{D}_{a}\end{array}\right]$,

$\beta_{e}=\left[\begin{array}{ll}\mathbf{I}_{e} & \mathbf{J}_{e}\end{array}\right], \Lambda_{3}=\frac{P_{S}}{n_{B_{2}} N_{S}} \oplus \frac{P_{S}}{n_{B_{1}} N_{S}}$ and $\Lambda_{4}=\frac{P_{S}}{n_{B_{2}} N_{E}} \oplus \frac{P_{P}}{n_{B_{1}} N_{E}}$.

$$
\begin{gathered}
C_{P_{\text {mast }}^{\text {Sec }}}=\max _{Q_{X}} \log _{2}\left\{\frac{\left(\mathbf{I}_{n_{P}}+\min _{1 \leq a \leq P} \Theta_{a} \Lambda_{1} \Theta_{a}^{\dagger}\right)\left(\mathbf{I}_{n_{E}}+\frac{P_{S B S}}{n_{B_{2}} N_{P B S}} \max _{1 \leq e \leq K} \mathbf{J}_{e} \mathbf{J}_{e}^{\dagger}\right)}{\left(\mathbf{I}_{n_{P}}+\frac{P_{S B S}}{n_{B_{2}} N_{P B S}} \min _{1 \leq a \leq P} \mathfrak{G}\right)\left(\mathbf{I}_{n_{E}}+\max _{1 \leq e \leq K} \Omega_{e} \Lambda_{2} \Omega_{e}^{\dagger}\right)}\right\} \text { bits/sec/Hz (11) } \\
C_{S_{\text {mast }}^{\text {Sec }}}=\max _{Q_{S}} \log _{2}\left\{\frac{\left(\mathbf{I}_{n_{S}}+\min _{1 \leq b \leq S} \alpha_{b} \Lambda_{3} \alpha_{b}^{\dagger}\right)\left(\mathbf{I}_{n_{E}}+\frac{P_{S}}{n_{B_{2}} N_{P}} \max _{1 \leq e \leq K} \mathbf{I}_{e} \mathbf{I}_{e}^{\dagger}\right)}{\left(\mathbf{I}_{n_{S}}+\frac{P_{S}}{n_{B_{2}} N_{P}} \min _{1 \leq b \leq S} \mathbf{D}_{b} \mathbf{D}_{b}^{\dagger}\right)\left(\mathbf{I}_{n_{E}}+\max _{1 \leq e \leq K} \beta_{e} \Lambda_{4} \beta_{e}^{\dagger}\right)}\right\} \text { bits/sec/Hz (12) }
\end{gathered}
$$

\section{Transmission with Precoding Relay}

In this section, the SMC has been derived with different precoding relays. For this purpose, it is considered that, the PBS, SBS and precoding relay transmit simultaneously to the PRs and SRs. Received signals at the ath PRs, bth SRs and eth eavesdroppers with the precoding relay are given by

$$
\begin{array}{r}
\mathbf{y}_{P_{a}}=\mathbf{H}_{a} \mathbf{x}_{1}+\mathbf{G}_{a} \mathbf{x}_{2}+\mathbf{U}_{a} \mathbf{s}+\mathbf{n}_{P_{a}} . \\
\mathbf{y}_{S_{b}}=\mathbf{B}_{b} \mathbf{x}_{2}+\mathbf{D}_{b} \mathbf{x}_{1}+\mathbf{V}_{b} \mathbf{s}+\mathbf{n}_{S_{b}} . \\
\mathbf{y}_{K_{e}}=\mathbf{I}_{e} \mathbf{x}_{1}+\mathbf{J}_{e} \mathbf{x}_{2}+\mathbf{W}_{e} \mathbf{s}+\mathbf{n}_{E_{e}} .
\end{array}
$$

Let, $\mathbf{s} \in \mathbb{C}^{n_{R} \times 1}$ denotes the transmitted precoded signal of the relay. The channel coefficients between precoding relay and PRs are $\mathbf{U}_{a} \in \mathbb{C}^{n_{P} \times n_{R}}$ and that between precoding relay and SRs are $\mathbf{V}_{b} \in \mathbb{C}^{n_{S} \times n_{R}}$. The channel coefficient between precoding relay and eavesdroppers are $\mathbf{W}_{e} \in \mathbb{C}^{n_{E} \times n_{R}}$.

In order to transmit estimated channel information and data, PBS and SBS communicate via precoding relay and receive a power scaling factor determined by the linear precoding relay. Power scaling factor is required to normalize the power at the receiving end of the antennas as the precoding matrices tend to increase the power of the transmitted signals of PBS and MBS. The precoded signals at the PBS and SBS are given by $\mathbf{x}_{1}=f \tau_{1} \mathbf{d}_{1}$ and $\mathbf{x}_{2}=f \tau_{2} \mathbf{d}_{2}$, respectively Where, $\mathbf{d}_{1} \in \mathbb{C}^{n_{P} \times 1}$ and $\mathbf{d}_{2} \in \mathbb{C}^{n_{S} \times 1}$ denote the data vectors of PBS and MBS, respectively. $\tau_{1} \in \mathbb{C}^{n_{B_{1}} \times n_{P}}$ and $\tau_{2} \in \mathbb{C}^{n_{B_{2}} \times n_{S}}$ represent the precoding matrices of PBS and SBS respectively, and $f$ denotes the power scaling factor provided by the precoding relay. Let $\mathbf{H}=\left[\begin{array}{llll}\mathbf{H}_{1} & \mathbf{H}_{2} & \cdots & \mathbf{H}_{P}\end{array}\right]^{\mathrm{T}}, \mathbf{G}=\left[\begin{array}{lllll}\mathbf{G}_{1} & \mathbf{G}_{2} & \cdots & \mathbf{G}_{P}\end{array}\right]^{\mathrm{T}}$, $\mathbf{B}=\left[\begin{array}{llll}\mathbf{B}_{1} & \mathbf{B}_{2} & \cdots & \mathbf{B}_{S}\end{array}\right]^{\mathrm{T}}, \quad \mathbf{G}=\left[\begin{array}{lllll}\mathbf{D}_{1} & \mathbf{D}_{2} & \cdots & \mathbf{D}_{S}\end{array}\right]^{\mathrm{T}} \quad \mathbf{U}=\left[\begin{array}{llll}\mathbf{U}_{1} & \mathbf{U}_{2} & \cdots & \mathbf{U}_{P}\end{array}\right]^{\mathrm{T}}$, $\mathbf{V}=\left[\begin{array}{llll}\mathbf{V}_{1} & \mathbf{V}_{2} & \cdots & \mathbf{V}_{S}\end{array}\right]^{\mathrm{T}}, \quad \mathbf{Z}_{P}=\left[\begin{array}{lllll}\mathbf{n}_{P_{1}} & \mathbf{n}_{P_{2}} & \cdots & \mathbf{n}_{P_{P}}\end{array}\right]^{\mathrm{T}}$ and $\mathbf{Z}_{S}=\left[\begin{array}{llll}\mathbf{n}_{S_{1}} & \mathbf{n}_{S_{2}} & \cdots & \mathbf{n}_{S_{S}}\end{array}\right]^{\mathrm{T}}$. The total received signals at the PRs and SRs can be written as

$$
\begin{array}{r}
\mathbf{y}_{P}=\mathbf{H} \mathbf{x}_{1}+\mathbf{G} \mathbf{x}_{2}+\mathbf{U s}+\mathbf{Z}_{P} . \\
\mathbf{y}_{S}=\mathbf{B} \mathbf{x}_{2}+\mathbf{D} \mathbf{x}_{1}+\mathbf{V s}+\mathbf{Z}_{S} .
\end{array}
$$


Let, $\Psi=\left[\begin{array}{cc}\mathbf{H} \tau_{1} & \mathbf{G} \tau_{2} \\ \mathbf{B} \tau_{1} & \mathbf{D} \tau_{2}\end{array}\right]$ is the channel cross-correlation matrix which includes the correlation elements between the antennas in PRs and SRs as well as the correlation between the cross channels, $\mathbf{Y}=\left[\begin{array}{ll}\mathbf{y}_{P} & \mathbf{y}_{S}\end{array}\right]^{\mathrm{T}}, \mathbf{Q}=\left[\begin{array}{ll}\mathbf{U} & \mathbf{V}\end{array}\right]^{\mathrm{T}}$ and $\mathbf{Z}=\left[\begin{array}{ll}\mathbf{Z}_{P} & \mathbf{Z}_{S}\end{array}\right]^{\mathrm{T}}$. Then Equation (16) and Equation (17) can be rewrite as

$$
\mathbf{Y}=f \Psi \mathbf{d}+\mathbf{Q s}+\mathbf{Z} \text {. }
$$

\subsection{SMC with ZF Precoding Relay}

The aim of the ZF precoding relay is zeroing the cross interference between the two systems (MBS and PBS) and to fulfill this aim a precoder has been designed at the $\mathrm{ZF}$ precoding relay according to the $\mathrm{ZF}$ criterion to cancel the mean square error (MSE) [15]. Assuming a restriction to the total average transmit power to unity and based on the precoded symbols with transmitted powers $P_{P}=\operatorname{tr}\left[\tau_{1} \tau_{1}^{\dagger}\right], \quad P_{S}=\operatorname{tr}\left[\tau_{2} \tau_{2}^{\dagger}\right] \quad$ and $\quad P_{R}=\operatorname{tr}\left[(\mathbf{I}-\Psi)^{\dagger} \vartheta^{-1}(\mathbf{I}-\Psi)\right] \quad$ where $\vartheta=\mathbf{Q Q}^{\dagger}$, the power scaling factor for ZF precoding relay, $f_{Z}$ employing Maximul Ratio Combining (MRC) diversity can be calculated as [1].

$$
f_{z}=\left\{\operatorname{tr}\left[\Upsilon^{\dagger} \vartheta^{-1} \Upsilon\right]+\operatorname{tr}[\kappa]+\operatorname{tr}[\mu]\right\}^{-\frac{1}{2}}
$$

where $\Upsilon=(\mathbf{I}-\Psi), \quad \kappa=\mathbf{H}_{a} \mathbf{H}_{a}^{\dagger}$ and $\mu=\mathbf{B}_{b} \mathbf{B}_{b}^{\dagger}$. Nullifying interferences using $\mathrm{ZF}$ precoding at the relay, the average received SNR per symbol at the PRs and SRs are given by $\bar{\gamma}_{p}=\frac{f_{z}^{2} P_{P}}{N_{P}}$ and $\bar{\gamma}_{s}=\frac{f_{z}^{2} P_{S}}{N_{S}}$, respectively. Therefore, the SMC at the PRs and SRs can be derived as

$$
\begin{gathered}
C_{\mathrm{mcast} \text { PR }}^{Z F}=\log _{2}\left(\frac{1+\frac{f_{z}^{2} P_{P}}{N_{P}}}{1+\frac{f_{z}^{2} P_{P}}{N_{E}+\max _{1 \leq a \leq P}\left\|\mathbf{J}_{e}\right\|^{2} P_{S}}}\right), \\
C_{\text {mcast }_{\mathrm{SR}}}^{Z F}=\log _{2}\left(\frac{1+\frac{f_{z}^{2} P_{S}}{N_{S}}}{1+\frac{f_{z}^{2} P_{S}}{N_{E}+\max _{1 \leq e \leq K}\left\|\mathbf{I}_{e}\right\|^{2} P_{P}}}\right),
\end{gathered}
$$

where $\frac{f_{z}^{2} P_{P}}{N_{E}+\max _{1 \leq e \leq K}\left\|\mathbf{J}_{e}\right\|^{2} P_{S}}$ denotes the received signal-to-interference plus noise ratio (SINR) at the eavesdroppers due the transmit signal of PBS. Similarly, $\frac{f_{z}^{2} P_{S}}{N_{E}+\max _{1 \leq e \leq K}\left\|\mathbf{I}_{e}\right\|^{2} P_{P}}$ denotes SINR at the eavesdroppers due the transmit signal of SBS.

\subsection{SMC with SP Relay}

For some specific symbol combinations which are known as green signal energy, interference existing in the communication system can be added to the desired 
symbols which improves the SNR at the receiver. Let the generic interference caused to $j$ th receive antenna from the $i$ th transmit antenna be $\lambda_{i, j}=d_{i} \rho_{i, j}$, where $d_{i}$ denotes the interfering signal and $\rho_{i, j}$ is the $i$, $j$ th correlation element in matrix $\Psi . \rho_{i, j}$ is said to be constructive interference if the real and imaginary parts of $\rho_{i, j}$ have the same sign as the real and imaginary parts of the desired symbol, respectively. Let $\xi_{R}$ and $\xi_{I}$ respectively denote the real and imaginary parts of the constructive interference. Then, we have

$$
\xi_{R}=\operatorname{Re}\left(\lambda_{i, j}\right) \operatorname{Re}\left(d_{j}\right)>0 \text { and } \xi_{I}=\operatorname{Im}\left(\lambda_{i, j}\right) \operatorname{Im}\left(d_{j}\right)>0,
$$

where $\operatorname{Re}(x)$ and $\operatorname{Im}(x)$ denote the real and imaginary parts of the complex number $x$. The correlation elements are beneficial to the received symbols? energy, when Equation (22) is satisfied. This positive interference power can be predicted and characterized using the knowledge of data and channels at the relay transmitter. Hence, as discussed in [1], precoder at the SP relay first forms a constructive correlation matrix $\beta$ as follows that contains the elements in $\Psi$ which fulfills the condition of constructive interference and removes the elements that creates destructive interference.

$$
[\beta]_{i, j}= \begin{cases}\rho_{i, j} & \text { if } \xi_{R}>0 \text { and } \xi_{I}>0 \\ 0 & \text { if } \xi_{R}<0 \text { and } \xi_{I}<0\end{cases}
$$

Following the similar procedure of previous section, the transmission power of relay can be formulated as $P_{R}=\operatorname{tr}\left[(\beta-\Psi)^{\dagger} \vartheta^{-1}(\beta-\Psi)\right]$, and the corresponding scaling factor for $\mathrm{SP}$ is given by

$$
f_{s}=\left\{\operatorname{tr}\left[\Theta^{\dagger} \vartheta^{-1} \Theta\right]+\operatorname{tr}\left[\left(\tau_{1} \tau_{1}^{\dagger}\right)^{-1}\right]+\operatorname{tr}\left[\left(\tau_{2} \tau_{2}^{\dagger}\right)^{-1}\right]\right\}^{-\frac{1}{2}},
$$

where $\Theta=(\beta-\Psi)$. Using the scaling factor of (24), the SMC at the PRs and SRs are respectively given by

$$
\begin{gathered}
C_{\text {mcast } \mathrm{pR}}^{S P}=\log _{2}\left(\frac{1+\frac{f_{s}^{2} P_{P} \mathbf{d}^{\dagger} \beta_{P}^{\dagger} \beta_{P} \mathbf{d}}{N_{P} n_{T}}}{1+\frac{f_{s}^{2} P_{P} \mathbf{d}^{\dagger} \beta_{p}^{\dagger} \beta_{P} \mathbf{d}}{\left(N_{e}+\max _{1 \leq e \leq K}\left\|\mathbf{J}_{e}\right\|^{2} P_{S}\right) n_{T}}}\right), \\
C_{\text {mcast } \mathrm{SR}}^{S P}=\log _{2}\left(\frac{1+\frac{f_{S}^{2} P_{S} \mathbf{b}^{\dagger} \beta_{S}^{\dagger} \beta_{S} \mathbf{b}}{N_{S} n_{T}}}{1+\frac{f_{s}^{2} P_{S} \mathbf{d}^{\dagger} \beta_{S}^{\dagger} \beta_{S} \mathbf{d}}{\left(N_{e}+\max _{1 \leq e \leq K}\left\|\mathbf{I}_{e}\right\|^{2} P_{P}\right) n_{T}}}\right),
\end{gathered}
$$

where $n_{T}=n_{b_{1}}+n_{b_{2}}$.

\subsection{SMC with PAP Relay}

Instead of zeroing the elements of channel cross correlation matrix $\Psi$, which create destructive interference, the resulting interference can be converted to constructive interference by correcting the phase of transmitted symbols and 
equivalently rotating the angle of correlation between them. The relative phase $\theta_{i, j}$ can be expressed as $\theta_{i, j}=d_{j} \frac{\operatorname{conj}\left(d_{i} \rho_{i, j}\right)}{\left|\rho_{i, j}\right|}$, where $\operatorname{conj}(x)$ and $|x|$ represent the conjugate and magnitude of complex number $x$. the amplitude of the rotated correlation remains unaltered due to the assumption that $\left|d_{i}\right|=1$ and hence $\left|\theta_{i, j}\right|=1$. The precoder at the relay is designed considering the phase alignment criterion to null the mean square error. Therefore, the precoder at the relay at first forms a modified correlation matrix $\alpha$ as follows stated in [1],

$$
[\alpha]_{i, j}=\rho_{i, j} \theta_{i, j} .
$$

The transmission power of the relay is formulated as $P_{R}=\operatorname{tr}\left[(\theta-\Psi)^{\dagger} \vartheta^{-1}(\theta-\Psi)\right]$, and the corresponding power scaling factor is given by

$$
f_{p}=\left\{\operatorname{tr}\left[\Gamma^{\dagger} \vartheta^{-1} \Gamma\right]+\operatorname{tr}\left[\left(\tau_{1} \tau_{1}^{\dagger}\right)^{-1}\right]+\operatorname{tr}\left[\left(\tau_{2} \tau_{2}^{\dagger}\right)^{-1}\right]\right\}^{-\frac{1}{2}},
$$

where $\Gamma=(\alpha-\Psi)$. Using the power scaling factor of (28), the SMC at the PRs and SRs are respectively derived as

$$
\begin{gathered}
C_{\text {mcast }_{\mathrm{PR}}}^{\text {PAP }}=\log _{2}\left(\frac{1+\frac{f_{P}^{2} P_{P} \mathbf{d}^{\dagger} \theta_{P}^{\dagger} \theta_{P} \mathbf{d}}{N_{P} n_{T}}}{1+\frac{f_{p}^{2} P_{P} \mathbf{d}^{\dagger} \Phi_{P}^{\dagger} \Phi_{P} \mathbf{d}}{\left(N_{e}+\max _{1 \leq e \leq K}\left\|\mathbf{J}_{e}\right\|^{2} P_{2} S\right) n_{T}}}\right) . \\
C_{\text {masts }_{\mathrm{SR}}}^{\text {PAP }}=\log _{2}\left(\frac{1+\frac{f_{P}^{2} P_{S} \mathbf{d}^{\dagger} \Phi_{S}^{\dagger} \Phi_{S} \mathbf{d}}{N_{2} n_{T}}}{1+\frac{f_{p}^{2} P_{S} \mathbf{d}^{\dagger} \Phi_{S}^{\dagger} \Phi_{S} \mathbf{d}}{\left(N_{e}+\max _{1 \leq e \leq K}\left\|\mathbf{I}_{e}\right\|^{2} P_{P}\right) n_{T}}}\right) .
\end{gathered}
$$

\section{SOP}

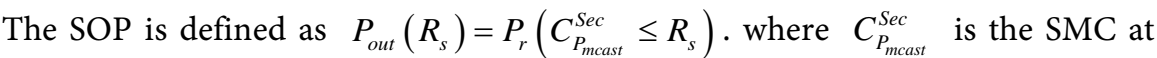
the PRs and $R_{s}$ is a target threshold. The SMC at the PRs with ZF precoding, SP and PAP has been derived in section IV and using those equations SOP for ZF precoding, SP and PAP relays can be analyzed.

\section{Numerical Results}

In this section, the effect of interference on the SMC at the PRs and SRs has been investigated by simulating the equations which was derived in Section III, IV and V. In order to obtain the simulation results for the SMC and the SOP for multicasting the simulation environments have been developed in MATLAB by generating and averaging the Rayleigh fading channels with 110,000 realizations. Figure 2 and Figure 3 show the effect of interference on the SMC of PRs and SRs, respectively as a function of transmit power of base stations. It is noticed 


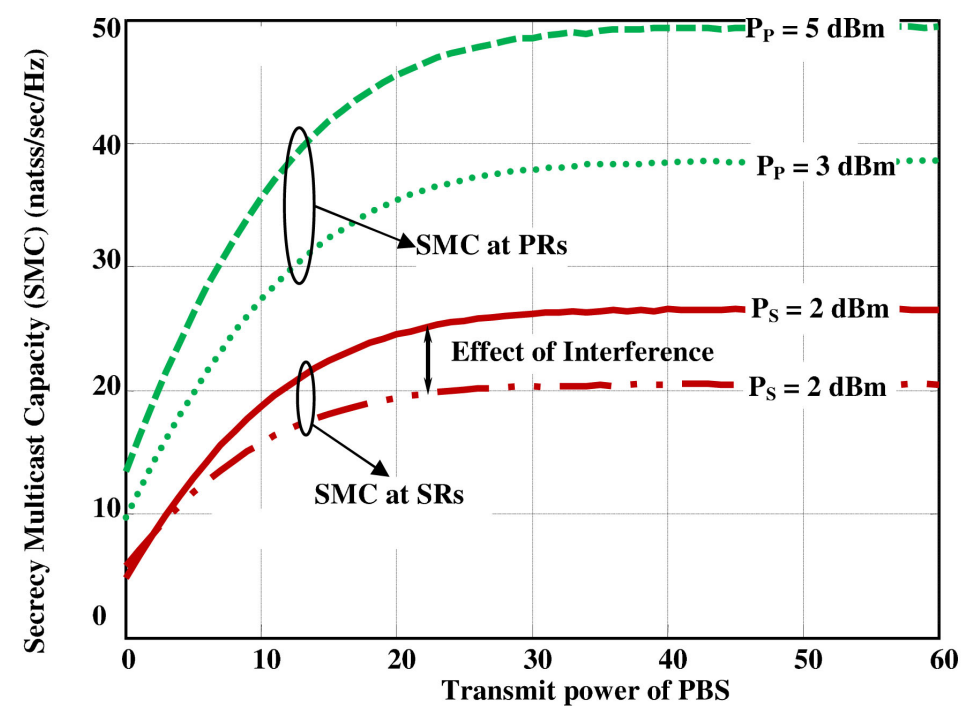

Figure 2. Effect of interference on the SMC at SRs for selected values of transmitted power of PBS.

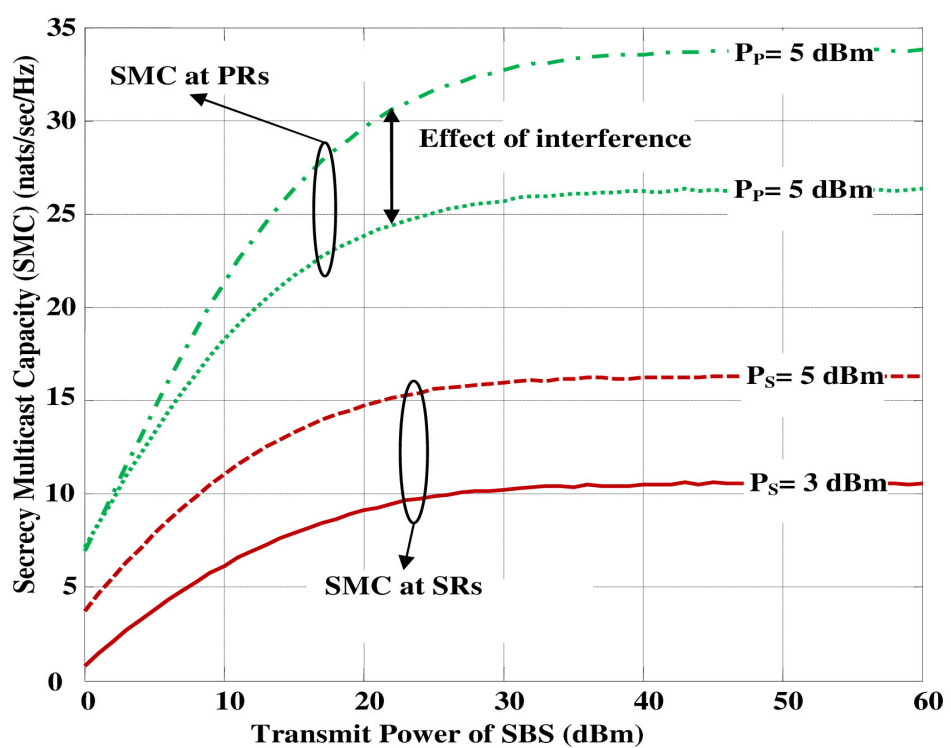

Figure 3. Effect of interference on the SMC at PRs for selected values transmitted power of SBS.

that if the transmit power of PBS is increased (in Figure 2) the SMC at the PRs increases but the SMC at the SRs decreases due to the effect of interferences caused by the transmitted signals of PBS. Similar scenario is observed in the case of SMC at the PRs. If the transmit power of SBS increases (in Figure 3) the SMC at the SRs increases but the SMC at the PRs decreases due to the effect of interferences caused by the transmitted signals of SBS.

Then the effect of using different types of precoding relays (ZF, SP and PAP) on the SMC at the PRs has been investigated to nullify the interference power as well as to use the constructive and destructive interference power. Figure 4 depicts the simulation result of SMC at the PRs as a function of SNR in (dB) which 
is obtained from the derived equations (equation no 20, 25 and 29) to compare the performance of employing $\mathrm{ZF}, \mathrm{SP}$ and PAP at the relay. It is observed from Figure 4, that using PAP at the relay enhances the SMC in a larger scale compared to SP and ZF. This is because, ZF precoding relay only cancels out the interference while SP uses the constructive interference energy as an additional source of energy which increases the SNR at the PRs whereas PAP uses both constructive and destructive interference power by rotating the phase angle of destructive interference.

Figure 5 describes the effect of using ZF precoding, SP and PAP at the relay on the SOP as a function of target rate in nats/ $\mathrm{Hz}$. It is observed from Figure 5 that SOP at the PRs is better for PAP than that of other two precoding relays ( $\mathrm{ZF}$ precoding and SP).

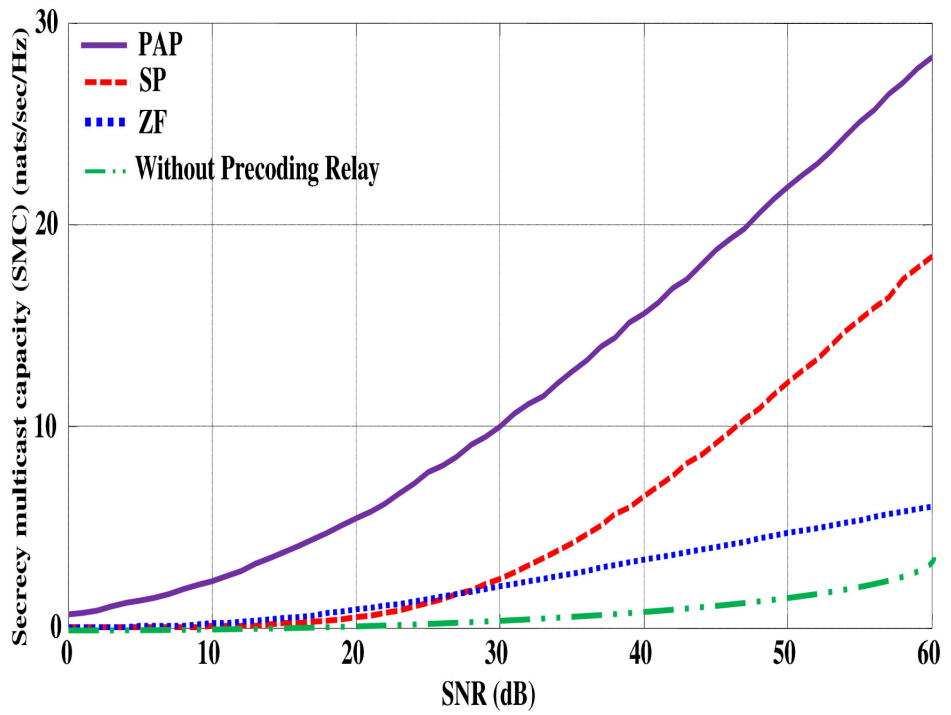

Figure 4. Comparison of SMC at the PRs employing ZF precoding, SP and PAP at the relay.

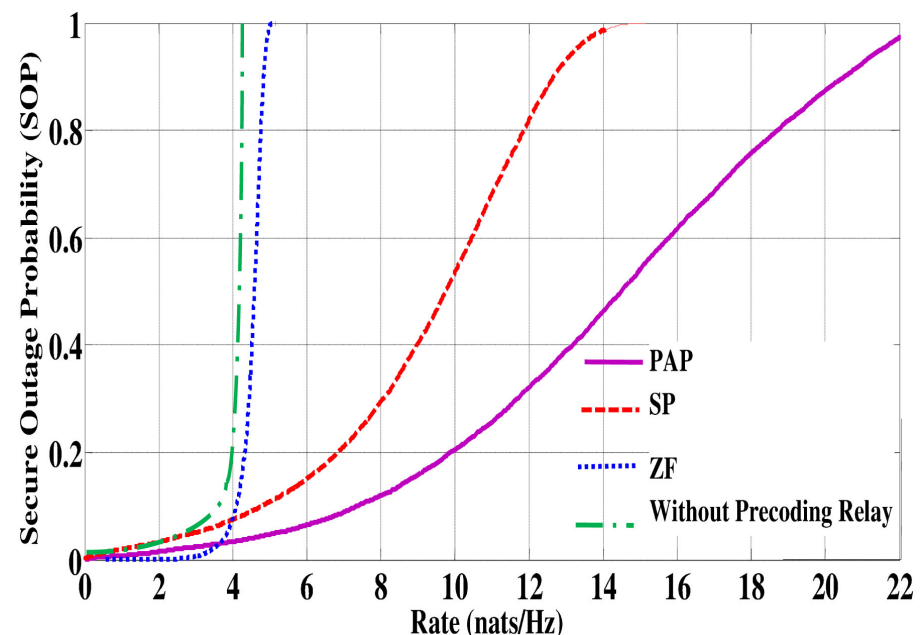

Figure 5. Comparison of SOP at the PRs employing ZF precoding, SP and PAP at the relay. 


\section{Conclusion}

In this paper, a scheme has been proposed to use the destructive interference power in enhancing the SMC for MIMO CRNs. Based on our formulation and observation of numerical results, it is concluded that, although interference reduces the SMC, it is possible to enhance the system performance employing precoding technique at the relay. In the case of ZF precoding at the relay, the effect of interference is canceled while in the case of SP at the relay, constructive interference power can be used as an additional source of energy to enhance the SMC and SOP without increasing transmit signal power. In the case of PAP at the relay, the destructive part of interference advances the SMC and SOP one step ahead than the former two precoding techniques (ZF precoding and SP).

\section{Acknowledgements}

This work was supported by the Research Support and Publication Division of University Grant Commission, Bangladesh.

\section{Conflicts of Interest}

The authors declare no conflicts of interest regarding the publication of this paper.

\section{References}

[1] Masouros, C. and Ratnarajah, T. (2012) Interference as a Source of Green Signal Power in Cognitive Relay-Assisted Co-Existing MIMO Wireless Transmissions. IEEE Transactions on Communications, 60, 525-536. https://doi.org/10.1109/TCOMM.2011.112811.100734

[2] Sultana, R., Sarkar, M.Z.I., Faisal, K.N., Giti, J.E. and Ahmed, F.R.S. (2014) Enhancing Wireless Capacity in Heterogeneous Networks with Interference Power. 201417 th International Conference on Computer and Information Technology, Dhaka, Bangladesh, 22-23 December 2014, 458-461. https://doi.org/10.1109/ICCITechn.2014.7073076

[3] Hong, X., Chen, Z., Wang, C.-X. Vorobyov, S.A. and Thompson, J.S. (2009) Interference Cancelation and Management Techniques. IEEE Vehicular Technology Magazine, 4, 76-84. https://doi.org/10.1109/MVT.2009.934672

[4] Zou, Y., Champagne, B., Zhu, W.-P. and Hanzo, L. (2014) Relay-Selection Improves the Security-Reliability Trade-off in Cognitive Radio Systems. IEEE Transactions on Communication, 63, 215-228. https://doi.org/10.1109/TCOMM.2014.2377239

[5] Zhong, S. and Yao, H. (2013) Towards Cheat-Proof Cooperative Relay for Cognitive Radio Networks. IEEE Transactions on Parallel and Distributed Systems, 25, 2442-2451. https://doi.org/10.1109/TPDS.2013.151

[6] Jeon, H., McLaughlin, S.W., Kim, I.-M. and Ha, J. (2015) Secure Communications with Untrusted Secondary Nodes in Cognitive Radio Networks. IEEE Transactions on Wireless Communications, 13, 1790-1805. https://doi.org/10.1109/TWC.2013.021214.130089

[7] Pu, D. and Wyglinski, A.M. (2014) Primary-User Emulation Detection Using Database-Assisted Frequency-Domain Action Recognition. IEEE Transactions on Vehicular Technology, 63, 4372-4382. https://doi.org/10.1109/TVT.2014.2316831 
[8] Wang, C. and Wang, H.-M. (2014) Primary-User Emulation Detection Using Database-Assisted Frequency-Domain Action Recognition. IEEE Transactions on Information Forensics and Security, 9, 1814-1827. https://doi.org/10.1109/TIFS.2014.2356339

[9] Zou, Y., Wang, X. and Sen, W. (2013) Physical-Layer Security with Multiuser Scheduling in Cognitive Radio Networks. IEEE Transactions on Wireless Communications, 13, 5103-5113. https://doi.org/10.1109/TCOMM.2013.111213.130235

[10] Du, J., Xiao, M., Skoglund, M. and Medard, M. (2013) Wireless Multicast Relay Networks with Limited-Rate Source-Conferencing. IEEE Journal on Selected Areas in Communications, 31, 1390-1401. https://doi.org/10.1109/JSAC.2013.130804

[11] Ghaznavi, A. and Razavizadeh, S.M. (2009) A Precoding Technique for Joint Relaying and Broadcasting in Cognitive Radio Networks. 2009 IEEE Pacific Rim Conference on Communications, Computers and Signal Processing, Victoria, 23-26 August 2009, 383-387. https://doi.org/10.1109/PACRIM.2009.5291340

[12] Chen, Z., Wang, C.-X., Hong, X., Thompson, J., Vorobyov, S.A., Zhao, F., Xiao, H. and Ge, X. (2011) Interference Mitigation for Cognitive Radio MIMO Systems Based on Practical Precoding. Physical Communication, 9, 308-315.

[13] Ghebretensae, Z., Harmatos, J. and Gustafsson, K. (2010) Mobile Broadband Backhaul Network Migration from TDM to Carrier Ethernet. IEEE Communications Magazine, 48, 102-109. https://doi.org/10.1109/MCOM.2010.5594684

[14] Sridharan, S., Vishwanath, S., Jafar, S.A. and Shamai, S. (2008) On the Capacity of Cognitive Relay Assisted Gaussian Interference Channel. 2008 IEEE International Symposium on Information Theory, Toronto, Canada, 6-11 July 2008, 549-553. https://doi.org/10.1109/ISIT.2008.4595046

[15] Vojeie, B.R. and Jang, W.M. (1998) Transmitter Precoding in Synchronous Multiuser Communications. IEEE Transactions on Communication, 46, 1346-1355. https://doi.org/10.1109/26.725312 